\title{
Meiosis in male and female Trichosurus vulpecula (Marsupialia)
}

\author{
D. L. Hayman* and \\ J. C. Rodger†
}

\author{
* Department of Genetics, University of Adelaide, \\ South Australia 5000. \\ † Department of Biological Sciences, University of \\ Newcastle, N.S.W. 2308.
}

At meiosis in female Trichosurus vulpecula there are fewer chiasmata per cell and fewer interstitial chiasmata than are observed in male $T$. vulpecula. These observations suggest that recombination in the female will be less than that observed in the male; however, this difference between the sexes will not be as great as that previously described in other marsupial species.

\section{INTRODUCTION}

Genetical and cytological studies of the small marsupial Sminthopsis crassicaudata (Dasyuridae) have demonstrated a novel difference between the sexes in both genetic recombination and chiasma distribution (Bennett et al., 1986). Analyses of the segregation of genes in two autosomal linkage groups showed that recombination was very much higher in males than in females from whom few recombinant progeny were recovered. The distribution of chiasmata at meiosis in the two sexes provided an explanation for these observations. In females the mean chiasma frequency per cell was slightly lower than that found in males, and the chiasmata showed pronounced and possibly exclusive distal localisation. In males half of the chiasmata were clearly interstitial.

This substantial difference in recombination between the sexes is not only much greater than that usually observed in eutherian mammals, but also is in the opposite direction to those differences generally recorded. In eutherian mammals it is usual for the homogametic female to have a higher recombination frequency than the heterogametic male (Dunn and Bennett, 1967.

The possibility that this difference may be common in, and perhaps general to all marsupials was suggested by the observation of Hayman et al. (1988). A study of meiosis in both sexes of Monodelphis domestica (Didelphidae), a South American marsupial, showed essentially the same differences in chiasma frequency and distribution as observed in $S$. crassicaudata. The present day elements of the Australian and South American marsupial faunas are thought to have a common origin, probably in North America. The presence of this difference in the only representatives studied in both faunas suggests that it was present before the time of divergence of these groups.

It is of considerable interest to test whether this difference exists in other marsupial lineages. The choice of species in which such a study may be made is limited. Attempts to culture marsupial oocytes have failed, thus observation of the informative stages of meiosis is effectively restricted to polyovulatory species in which a number of oocytes mature at the same time, or to monovulatory species in which multiple ovulation can be induced. The recent successful induction of superovulation in the normally monovulatory and commonly occurring brush-tailed possum Trichosurus vulpecula (Phalangeridae) (Rodger and Mate, 1988) offered an opportunity to investigate a marsupial from a previously unstudied group.

\section{MATERIALS AND METHODS}

Ovaries of mature, usually lactating, female possums collected in the Adelaide area were induced to undergo multiple follicle development and oocyte maturation by a combined gonadotrophin/synthetic releasing hormone treatment based on the protocol of Rodger and Mate (1988). Pregnant mare serum gonadotrophin 
(PMSG) (Folligon; Intervert, Holland) was used at either 7.5 or 10 i.u. followed 2.5 or 3 days later by three injections (Fertagyl; Intervet, Holland). Six to $18.75 \mathrm{~h}$ after the first GnRH injection the animals were killed by barbiturate overdose (Lethabarb; Arnolds, U.K.) and the ovaries removed.

Ovaries were examined using a dissecting microscope and the maximum diameter of the protruding follicles measured to the nearest
$0 \cdot 1 \mathrm{~mm}$ with vernier callipers. Oocytes were collected into culture medium, from all follicles greater than $2.5 \mathrm{~mm}$ in diameter, by pricking each follicle with a fine hypodermic needle. The surface of the zona pellucida of recovered oocytes were partly or completely shrouded by a layer of residual granulosa cells. However, these were easily removed from oocytes undergoing nuclear maturation, by passing the oocyte repeatedly through a fine glass pipette (diameter slightly less than the
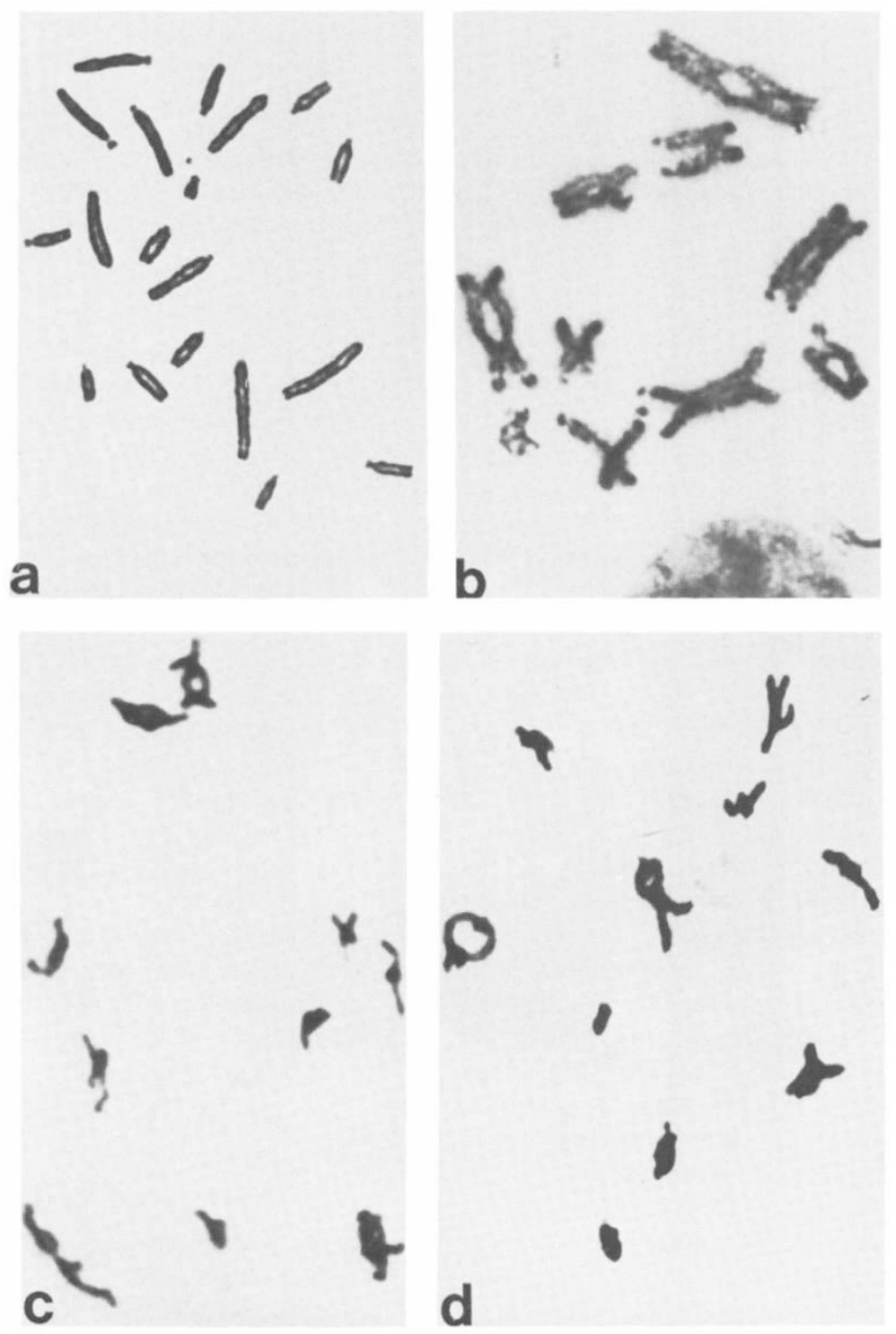

Figure 1 (a) mitotic metaphase of male T. vulpecula. The Y chromosome is the small dot-like element; (b) diplotene of meiosis in male T. vulpecula showing interstitial chiasmata; (c) and (d) diplotene/diakinesis in female T. vulpecula showing some interstitial chiasmata present. 
diameter of the oocyte) controlled by a mouth operated suction tube.

Oocytes were placed in $0.075 \mathrm{M} \mathrm{KCl}$ for $1 \frac{1}{2}$ minutes and then fixed and stained following the method of Tarkowski (1966). Oocytes exposed to $\mathrm{KCl}$ for more than two minutes become too fragile for satisfactory preparations. Passage of the nuclei of oocytes through the meiotic stages up to metaphase is very quick, and at metaphase the chromosomes are confined to a small volume of the nucleus and are too condensed to give information about chiasmata.

\section{RESULTS}

Oocytes coming out of the dictyate stage through to fully compacted metaphase I were obtained at a mean of $16 \cdot 2 \mathrm{~h}(\mathrm{SEM} 0 \cdot 52, n=15)$ after the first $\mathrm{GnRH}$ injection. The mean maximum diameter of the follicles from which these oocytes were obtained was $3.4 \mathrm{~mm}$ (SEM 1.14, $n=15$ ).

The chromosome number of $T$. vulpecula is $2 n=20$ and the chromosomes are, with one exception, acrocentrics with very short arms (see fig. 1(a)). A total of eight cells derived from six female animals gave data on chiasma frequency and distribution. The average chiasma frequency per cell is 12.6 and the range is from 11-14. There was an average of six well-defined interstitial chiasmata present in each cell. There is no evidence of distal localisation of all chiasmata (see fig. 1(c) and (d)).

Meiosis in male T. vulpecula collected from the same area as the females has been studied in some detail (Sharp and Hayman, 1985). In a total of 69 wild caught animals the average number of chiasmata per cell ranged from $14 \cdot 15$ to 21.85 with a mean overall of $18 \cdot 14 \pm 1.96$ chiasmata. There were on average 10.8 clearly interstitial chiasmata per cell (see fig. 1(b)).

A comparison of the frequency of interstitial chiasmata in the eight oocytes with those present in fifteen meiotic cells from two males is shown in fig. 2. The frequency of interstitial chiasmata is positively correlated with the total chiasmata frequency over both populations of cells.

\section{DISCUSSION}

The cytological data suggests that the frequency of recombination which will be found in Trichosurus females will, in general, be less than that found in Trichosurus males. Thus the difference between the sexes which has been
14

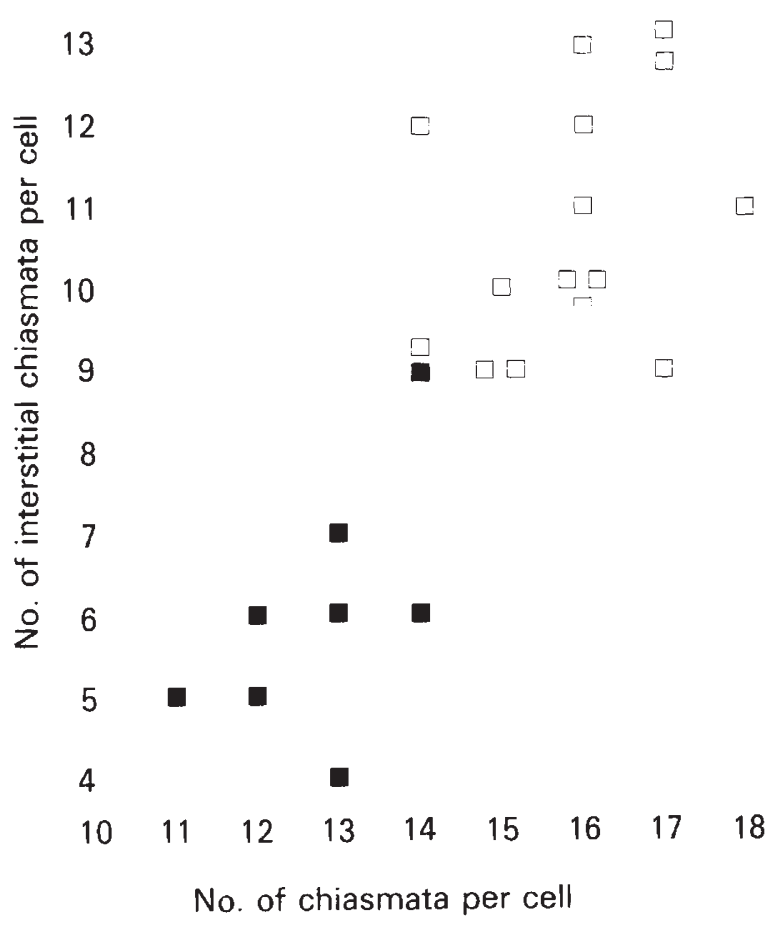

Figure 2 The relationship between number of interstitial chiasmata per cell and the total number of chiasmata in that cell in females (solid symbols) and males (hollow symbols) of $T$. vulpecula (see text for details). No chiasmata occur between the $\mathrm{X}$ and $\mathrm{Y}$ chromosome in the male.

demonstrated in Sminthopsis and in Monodelphis is maintained in the third species of marsupial to be studied. However, the differences between the sexes in Trichosurus would be anticipated to be less extreme than those in the other species. Further, if there are differences in chiasma localisation between the sexes there could well be regions of the linkage map in which recombination frequency in the female is higher than in the male.

The extreme differences observed in Sminthopsis are the result of the virtual elimination of interstitial chiasmata in the female with only a small reduction in the total chiasmata present compared to those found in the male (Bennett et al., 1986). A different cytological mechanism apparently operates in Trichosurus. Here the reduction in the total number of chiasmata found in the female is associated with the reduction in interstitial chiasmata. Thus there is a modulation of the level of recombination.

There is no explanation at present for the differences in recombination found between the 
sexes when, as is usual, the heterogametic sex has the lower value. The fact that marsupials have apparently adopted a variant strategy does not, of itself, offer a reason. This paper suggests that the marsupial variation on this theme will differ in its extent between species, just as in the more usual situation there are also variations in the extent of the difference between the sexes. It may be significant that the most extreme differences between the sexes in marsupials are shown by species which normally produce a number of offspring at each birth.

Cano and Santos (in press) have reported on the differences found between the sexes in both chiasma frequency and distribution in six grasshopper species. They find higher chiasma frequencies in the male of all but one of the species and no difference in the sixth; there are more interstitial and distal chiasmata in the females of all six species. The authors point out that this difference is not consistently observed in grasshoppers, even between closely related species, which "makes it difficult to envisage the adaptive value, if any, of these changes in recombination". While not necessarily agreeing with this conclusion, it is clear that additional data on this metric need to be obtained from other marsupial species.
Acknowledgements We express our appreciation of the financial support from the Mark Mitchell Fund; and the assistance of Mr Allen McNeil in collecting animals. The conduct of the research programme had the approval of the University of Adelaide Animal Ethics Committee.

\section{REFERENCES}

BENNETT, J. H., HAYMAN, D. L. AND HOPE, R. M. 1986. Novel sex difference in linkage values and meiotic chromosome behaviour in a marsupial. Nature, 233, 59-60.

CANO, M. I. AND SANTOS, J. L. Chiasma frequencies and distributions in gomphocerine grasshoppers: A comparative study between sexes. Heredity, (in press).

DUNN, L. C. AND BENNETT, DOROTHEA. 1967. Sex differences in recombination of linked genes in animals. Genet Res., 9, 211-220.

HAYMAN, D. L., MOORE, H. D. M. AND EVANS, E. P. 1988. Further evidence of novel sex differences in chiasma distribution in marsupials. Heredity, 61, 455-458.

RODGER, J. C. AND MATE, K. E. 1988. A PMSG/GnRH method for the superovulation of the monovulatory brushtailed possum (Trichosurus vulpecula). J. Reprod. Fert., 83, 885-891.

SHARP, P. J. AND HAYMAN, D. L. 1985. Variation in chiasma frequency in male Trichosurus vulpecula (Marsupialia: Mammalia). Genetica, 66, 145-150.

TARKOWSKI, A. K. 1966. An air drying method for chromosomal preparations from mouse eggs. Cytogenetics, 5, 394400. 